\title{
INDELseek: detection of complex insertions and deletions from next-generation sequencing data
}

\author{
Chun Hang Au', Anskar Y. H. Leung ${ }^{2}$, Ava Kwong ${ }^{3,4,5}$, Tsun Leung Chan ${ }^{1}$ and Edmond S. K. Ma ${ }^{\text {* }}$
}

\begin{abstract}
Background: Complex insertions and deletions (indels) from next-generation sequencing (NGS) data were prone to escape detection by currently available variant callers as shown by large-scale human genomics studies. Somatic and germline complex indels in key disease driver genes could be missed in NGS-based genomics studies.

Results: INDELseek is an open-source complex indel caller designed for NGS data of random fragments and PCR amplicons. The key differentiating factor of INDELseek is that each NGS read alignment was examined as a whole instead of "pileup" of each reference position across multiple alignments. In benchmarking against the reference material NA12878 genome ( $n=160$ derived from high-confidence variant calls), GATK, SAMtools and INDELseek showed complex indel detection sensitivities of $0 \%, 0 \%$ and 100\%, respectively. INDELseek also detected all known germline (BRCA1 and BRCA2) and somatic (CALR and JAK2) complex indels in human clinical samples $(n=8)$. Further experiments validated all 10 detected KIT complex indels in a discovery cohort of clinical samples. In silico semisimulation showed sensitivities of $93.7-96.2 \%$ based on 8671 unique complex indels in >5000 genes from dbSNP and COSMIC. We also demonstrated the importance of complex indel detection in accurately annotating BRCA1, BRCA2 and TP53 mutations with gained or rescued protein-truncating effects.
\end{abstract}

Conclusions: INDELseek is an accurate and versatile tool for complex indel detection in NGS data. It complements other variant callers in NGS-based genomics studies targeting a wide spectrum of genetic variations.

Keywords: Complex indel, Variant calling, Bioinformatics, Next-generation sequencing

\section{Background}

Complex insertions and deletions (indels) are a known class of genetic variation [1] associated with human diseases [2]. Simultaneous deletion and insertion of DNA fragments of different sizes lead to net change in length. No net change in length is also possible in case of contiguous or non-contiguous multiple-nucleotide variants (MNV). Compared with lower-throughput Sanger sequencing, analysis of next-generation sequencing data relies more on bioinformatics algorithms for automated variant calling. Of concern, recent studies revealed the shortcomings of state-of-the-art variant callers that might fail to detect somatic and germline complex indels $[3,4]$. Important mutations in key disease driver genes

\footnotetext{
* Correspondence: eskma@hksh.com

'Division of Molecular Pathology, Department of Pathology, Hong Kong

Sanatorium \& Hospital, Happy Valley, Hong Kong SAR

Full list of author information is available at the end of the article
}

could be missed in NGS-based genomics studies (e.g. somatic CALR complex indels in myeloproliferative neoplasms [5] and germline BRCA1/BRCA2 complex indels in hereditary breast and/or ovarian cancer [6]).

Pindel-C [3] was introduced to detect the complex indels missed by GATK [7] and VarScan [8] but the implementation was not yet publicly available. Amplicon Indel Hunter [9] and ScanIndel [10] were designed for those that led to $>5 \mathrm{bp}$ net change in length or softclipping, respectively. MAC [11] targeted MNV only by analyzing single nucleotide variant (SNV) calls of primary callers.

Here we present INDELseek, a software that directly calls somatic and germline complex indels from Sequence Alignment/Map (SAM/BAM) alignments regardless of net change in length. 


\section{Implementation}

The INDELseek algorithm was implemented as a single Perl script indelseek.pl that scans each NGS read alignment and identifies closely spaced substitutions, insertions or deletions in cis as potential complex indel regardless of net change in length. The only external dependency is SAMtools version 1.3 or above [12], which supports sequencing depth exceeding $8000 \mathrm{X}$ in case of deep amplicon sequencing. It was tested on both CentOS Linux 5.5 and Cray XC30 supercomputer (Extreme Scalability Mode) and can be run on the builtin Perl 5 installation of any Linux/Unix-like environment. Alignments of NGS reads in the de facto SAM/ BAM format [12] serve as input while any complex indel calls will be reported in variant call format (VCF) version 4.1 [13].

INDELseek was designed to identify complex indel(s) at single read level by examining each alignment as a whole (Fig. 1). In contrast, mainstream NGS variant callers examined each reference position across multiple alignments (also known as "pileup"), losing the haplotype information in case of multiple differences compared to reference. Mainstream NGS read aligners (e.g. BWAMEM) usually align complex indels as multiple mismatches, insertions and/or deletions (Concise Idiosyncratic
Gapped Alignment Report (CIGAR) operations M, I and D, respectively) clustered within a short window of reference/ read positions, which INDELseek was designed to detect. Since CIGAR operation M could represent either match or mismatch, it was first refined as operations $=$ for match and $\mathrm{X}$ for mismatch. INDELseek considers each window fulfilling all of these criteria as a complex indel call: (1) containing at least two $\mathrm{X}, \mathrm{I}$ and/or D operations that are at most $l$ nucleotides away from each other; (2) length at least two nucleotides. The parameter $l$ is five by default and is configurable through option-max_distance. For enhanced specificity, false positives can be marked and/or removed based on configurable filters of read base quality, allele frequency and allele depth.

INDELseek parameters were -skip_lowqual -skip_lowdepth-skip_lowaf-min_af 0.2 for germline $B R C A 1$ and $B R C A 2$ mutations, --skip_lowqual -skip_lowdepth -skip_lowaf -min_af 0.02 for somatic CALR, JAK2 and KIT mutations, and -skip_lowqual -skip_lowdepth -skip_lowaf -max_distance 10 -min_af 0.2 -min_depth 20 for NA12878 whole-genome sequencing (WGS) dataset. A single CPU core (2010 Intel Xeon X5660 2.8GHz) was measured to be capable to process 56,000 alignments per minute (275 bp MiSeq sequencing reads).

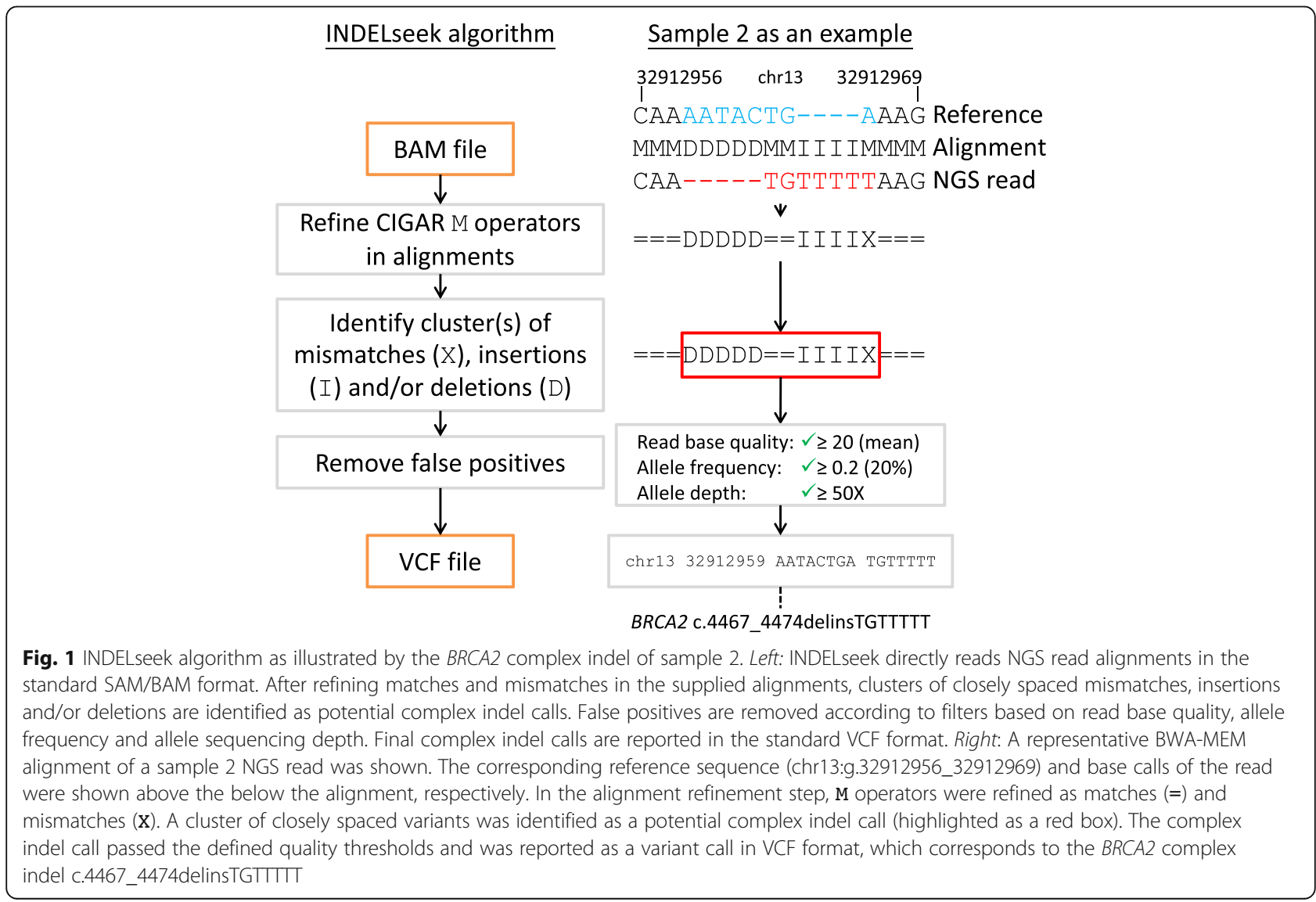




\section{Results}

We benchmarked complex indel detection performance of GATK, SAMtools and INDELseek using an external WGS dataset of the HapMap NA12878 genome (Illumina HiSeq 2000) and the corresponding high-confidence variant calls from the Genome in a Bottle (GIAB) Consortium [14]. Although the high-confidence variant calls did not comprise complex indels as individual calls, we observed clusters of closely spaced variants calls that appeared in cis in the alignments of individual sequencing reads. Accordingly, 160 such loci from GIAB calls were manually curated as putative complex indels (Additional file 1: Table S1) in the intersection (total length $27 \mathrm{Mb}$ ) of GIAB high confidence regions and Consensus Coding Sequence Project proteincoding sequences and $10 \mathrm{bp}$ intronic flanking regions [15]. We also observed closely spaced SNV that appeared in trans in the alignments and 26 such loci were manually curated as negative controls for complex indel detection (Additional file 1: Table S2). SAMtools and GATK did not call any complex indel from the putative GIAB complex indels ( 0 of 160) and negative controls (0 of 26), demonstrating $0 \%$ sensitivity and $100 \%$ specificity. The results were concordant with recent studies that complex indels were mostly missed by bioinformatics pipelines based on common variant callers [3, 4]. INDELseek called all putative GIAB complex indels (160 of 160) and did not call any from negative controls ( 0 of 26), demonstrating $100 \%$ sensitivity and $100 \%$ specificity (Table 1). All three types of complex indels resulting in net deletion of bases, no net change in length, or net insertion of bases were detected (Fig. 2a, b, c, respectively). In the context of complex indel detection, the whole-alignment-based approach of INDELseek was demonstrated to be superior to the conventional "pileup" approach of common variant callers.

Next, we tested INDELseek using two different NGS datasets of PCR amplicons (Table 1). INDELseek was applied to a hereditary breast and/or ovarian cancer (HBOC) panel dataset of 239 probands [6]. The 4-gene panel targeted germline mutations (Illumina MiSeq). Prior Sanger sequencing revealed that three of the probands carried a unique pathogenic complex indel (BRCA1 $n=1$ and $B R C A 2 n=2$ ) while remaining 236 probands were negative for complex indel. INDELseek detected all three complex indels (Table 2; Additional file 2: Figure S1-S3), demonstrating $100 \%$ sensitivity and $100 \%$ specificity. INDELseek was also applied to a myeloid neoplasm (MN) panel dataset of 23 samples [16]. The 54-gene panel targeted somatic mutations (Illumina MiSeq). From five samples known to carry a unique complex indel (CALR $n=4$ and JAK2 $n=1$ ), INDELseek detected all five complex indels (Table 2; Additional file 2: Figure S4-S8).

The general applicability of INDELseek in complex indel detection was further assessed using a wider spectrum of complex indels, which showed different
Table 1 Evaluation of INDELseek complex indel detection performance

\begin{tabular}{|c|c|c|c|}
\hline Dataset & $\begin{array}{l}\text { Sample count } \\
\text { and description }\end{array}$ & Sensitivity & Specificity \\
\hline \multicolumn{4}{|l|}{ Real NGS data } \\
\hline \multirow{3}{*}{$\begin{array}{l}\text { 1. Protein-coding and } \\
\text { flanking regions from } \\
\text { whole-genome } \\
\text { sequencing } \\
\text { (random fragments) }\end{array}$} & 1 (NA12878) & \multirow[t]{3}{*}{$100 \%$} & \multirow[t]{3}{*}{$100 \%$} \\
\hline & $\begin{array}{l}160 \text { putative } \\
\text { complex indels }\end{array}$ & & \\
\hline & $\begin{array}{l}26 \text { negative } \\
\text { control loci }\end{array}$ & & \\
\hline \multirow{3}{*}{$\begin{array}{l}\text { 2. Hereditary breast } \\
\text { and/or ovarian } \\
\text { cancer panel } \\
\text { (amplicons) }\end{array}$} & 239 & \multirow[t]{3}{*}{$100 \%$} & \multirow[t]{3}{*}{$100 \%$} \\
\hline & $\begin{array}{l}3 \text { positive samples } \\
(B R C A 1 n=1 \\
\text { BRCA2 } n=2)\end{array}$ & & \\
\hline & $\begin{array}{l}236 \text { negative } \\
\text { samples }\end{array}$ & & \\
\hline \multirow{3}{*}{$\begin{array}{l}\text { 3. Myeloid neoplasm } \\
\text { panel (amplicons) }\end{array}$} & 23 & \multirow[t]{3}{*}{$100 \%$} & \multirow[t]{3}{*}{$100 \%$} \\
\hline & $\begin{array}{l}5 \text { positive samples } \\
(C A L R n=4 \\
\text { JAK2 } n=1)\end{array}$ & & \\
\hline & $\begin{array}{l}18 \text { negative samples } \\
\text { (NA12878 and } 17 \\
\text { healthy controls) }\end{array}$ & & \\
\hline \multicolumn{4}{|c|}{ Semi-simulated data by engineering mutations to real NGS data } \\
\hline $\begin{array}{l}\text { 1. Whole-genome } \\
\text { sequencing } \\
\text { (random fragments) }\end{array}$ & $\begin{array}{l}8671 \text { collected from } \\
\text { COSMIC and dbSNP }\end{array}$ & $93.7 \%$ & N/A \\
\hline $\begin{array}{l}\text { 2. Hereditary breast } \\
\text { and/or ovarian } \\
\text { cancer panel } \\
\text { (amplicons) }\end{array}$ & $\begin{array}{l}237 \text { collected from } \\
\text { COSMIC and dbSNP }\end{array}$ & $96.2 \%$ & N/A \\
\hline $\begin{array}{l}\text { 3. Myeloid neoplasm } \\
\text { panel (amplicons) }\end{array}$ & $\begin{array}{l}576 \text { collected from } \\
\text { COSMIC and dbSNP }\end{array}$ & $94.6 \%$ & N/A \\
\hline
\end{tabular}

N/A Not applicable

combination of deletion and insertion lengths (375 combinations) and different gene context (>5000 genes). We collected 8671 unique complex indels from public databases dbSNP and COSMIC for semi-simulation by in silico engineering of complex indels in real NGS datasets. Base quality scores were kept unchanged or similar to flanking bases depending on the net gain in bases $(0$ or $\geq 1$, respectively). NGS data of NA12878, a BRCA1/BRCA2 complex indel-negative sample, and a healthy adult were selected for engineering from the WGS, HBOC and MN datasets, respectively. INDELseek demonstrated sensitivities of 93.7\% (8124 of 8671) for WGS, 96.2\% (228 of 237) for HBOC and 94.6\% (545 of 576) for MN (Table 1).

As a discovery cohort, INDELseek was applied to an additional MN panel dataset of 10 core-binding factor leukemia samples that were clinically predicted to be enriched for somatic mutations of KIT exon 8 [17]. A total of $10 \mathrm{KIT}$ in-frame complex indels were detected from six of the samples $(1-4$ complex indels per sample; Table 2) and verified by orthogonal validation experiments (Additional file 2: Figure S9-S14). 


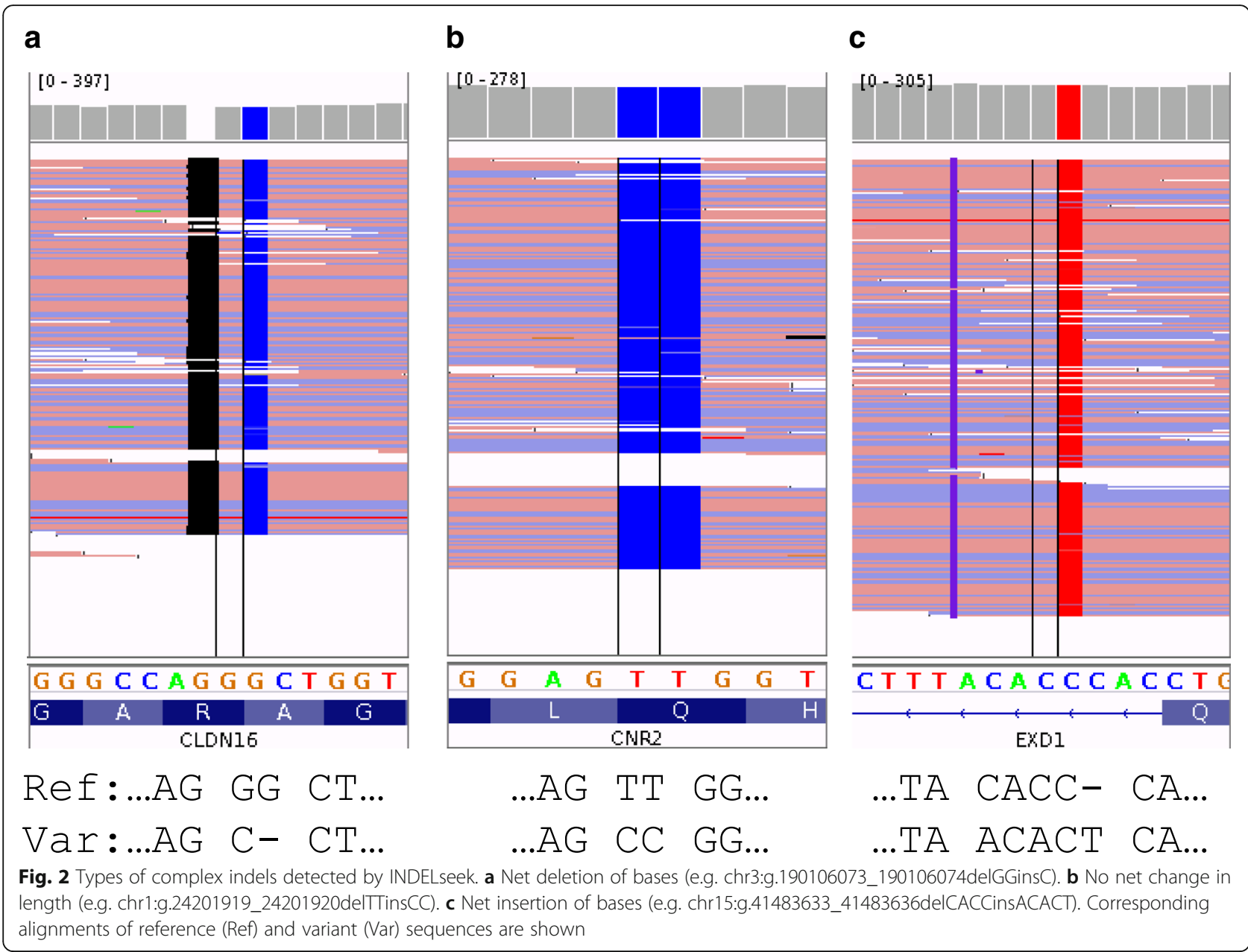

To demonstrate the importance of accurate complex indel detection in clinical settings, we focused on $127 \mathrm{MNV}$ in HBOC genes (part of semi-simulation collection) and compared their variant annotation results (Variant Effect Predictor) in two scenarios: (1) original MNV and (2) decomposing MNV into individual single-nucleotide variant for separate annotation, as if the MNV could not be called as a haplotype. Comparison revealed marked difference in $11(8.7 \%) \mathrm{MNV}$, which showed gained $(n=5)$ or rescued $(n=6)$ protein-truncating effects (Table 3$)$. Without accurate calling of complex indels, these MNV would become false negative or false positive pathogenic mutations, respectively. On the other hand, Variant Effect Predictor was tested to natively support complex indels called by INDELseek in VCF format.

\section{Conclusions}

This study showed that common variant callers fail to detect complex indels, a finding consistent with recent studies [3, 4]. We also demonstrated that if complex indels were called as individual variant calls (e.g. breaking down a single MNV to multiple SNV), the gained or rescued protein-truncating effects will be mis-interpreted. INDELseek was demonstrated as an accurate and versatile complex indel caller, which is compatible with somatic and germline genomics studies, NGS data of random fragments and PCR amplicons, and all three classes of complex indels (MNV, net insertion and net deletion). Since INDELseek was implemented as a single Perl script that directly reads SAM/BAM alignments and returns complex indel calls in VCF format, it can be readily incorporated into common bioinformatics workflows without any compilation and installation. INDELseek complements other common variant callers in academic and diagnostic NGSbased genomics studies.

\section{Methods}

\section{Benchmarking based on reference material}

High-confidence variants calls and chromosomal regions of NA12878 corresponded to the high-confidence genotype version 2.19 [14]. Closely spaced variant calls were identified by BEDTools version 2.19.1 [18] (parameters: merge $n-d$ 9). NA12878 200X whole genome sequencing dataset was retrieved from Illumina Platinum Genomes [19]. 
Table 2 Complex indels detected by INDELseek in human clinical samples

\begin{tabular}{|c|c|c|c|c|c|c|}
\hline Sample & Gene & Mutation & Allele frequency & $\begin{array}{l}\text { Sequencing } \\
\text { depth }(X)\end{array}$ & NGS method & $\begin{array}{l}\text { Orthogonal } \\
\text { validation }\end{array}$ \\
\hline \multicolumn{7}{|c|}{ Germline pathogenic mutations in hereditary breast and/or ovarian cancers } \\
\hline 1 & $B R C A 1$ & c.4046_4047delinsA p.Thr1349Lysfs*17 & $37.9 \%$ & 730 & * & $\dagger$ \\
\hline 2 & $B R C A 2$ & c.4467_4474delinsTGTTITT p.Lys1489Asnfs*15 & $74.9 \%$ & 1272 & * & $\dagger$ \\
\hline 3 & $B R C A 2$ & c.8400_8402delinsAAAA p.Phe2801Lysfs*11 & $33.6 \%$ & 4141 & * & $\dagger$ \\
\hline \multicolumn{7}{|c|}{ Somatic pathogenic mutations in myeloid neoplasms } \\
\hline 4 & CALR & c.1102_1136delinsT p.Lys368Trpfs*51 & $40.8 \%$ & 2274 & $\ddagger$ & $\dagger$ \\
\hline 5 & CALR & c.1154delAinsCTTGTC p.Lys385Thrfs*47 & $31.9 \%$ & 2998 & $\ddagger$ & $\dagger$ \\
\hline 6 & CALR & c.1129_1154delinsTGTC p.Lys377Cysfs*46 & $73.6 \%$ & 2159 & $\ddagger$ & $\dagger$ \\
\hline 7 & CALR & c.1118_1125delinsCTTG p.Asp373Alafs*56 & $15.3 \%$ & 3603 & $\neq$ & $\S$ \\
\hline 8 & JAK2 & c.1620_1627delinsGA p.lle540_Glu543delinsMetLys & $57.7 \%$ & 4629 & $\ddagger$ & $\dagger$ \\
\hline 9 & KIT & c.1248_1257delinsTTGG p.Thr417_Asp419delinsTrp & $39.0 \%$ & 11109 & $\neq$ & * \\
\hline \multirow[t]{4}{*}{10} & KIT & c.1248_1256delinsTTTCCG p.Thr417_Asp419delinsPheArg & $2.9 \%$ & 13724 & $\neq$ & * \\
\hline & KIT & c.1249_1258delinsGGATGGAACT p.Thr417_Arg420delinsGlyTrpAsnTrp & $3.3 \%$ & 13651 & $\ddagger$ & * \\
\hline & KIT & c.1250_1258delinsAACCTC p.Thr417_Asp419delinsLysPro & $11.9 \%$ & 13525 & $\ddagger$ & * \\
\hline & KIT & c.1251_1258delinsCTCCT p.Tyr418_Arg420delinsSerTrp & $2.1 \%$ & 13376 & $\neq$ & * \\
\hline \multirow[t]{2}{*}{11} & KIT & c.1250_1256delinsT p.Thr417_Asp419delinslle & $5.7 \%$ & 7326 & $\neq$ & $\S$ \\
\hline & KIT & c.1251_1257delinsAACA p.Tyr418_Asp419delinsThr & $2.2 \%$ & 7416 & $\neq$ & $\S$ \\
\hline 12 & KIT & c.1251_1256delinsGGG p.Tyr418_Asp419delinsGly & $2.7 \%$ & 14829 & $\neq$ & * \\
\hline 13 & KIT & c.1253_1258delinsCCG p.Tyr418_Arg420delinsSerGly & $40.7 \%$ & 68180 & $\neq$ & * \\
\hline 14 & KIT & c.1256_1257delinsGTCTA p.Asp419delinsGlyLeu & $17.9 \%$ & 19042 & $\ddagger$ & * \\
\hline
\end{tabular}

*Microfluidic PCR and MiSeq sequencing

†Sanger sequencing

¥Probe extension/ligation and MiSeq sequencing

§PCR fragment analysis

Table 3 Gained or rescued protein-truncating effect of complex indels

\begin{tabular}{|c|c|c|c|c|}
\hline \multirow[t]{2}{*}{ Gene } & \multirow[t]{2}{*}{ Genomic position } & \multirow[t]{2}{*}{ Multiple-nucleotide variants (MNV) } & \multicolumn{2}{|l|}{ Predicted protein change } \\
\hline & & & MNV called as a haplotype & MNV called as separate single-nucleotide variants \\
\hline \multicolumn{5}{|c|}{ Gained protein-truncating effect } \\
\hline$B R C A 2$ & 13:32914101-32914102 & c.5609_5610delTCinsAG & p.Phe1870* & p.Phe1870Tyr, p.Phe1870Leu \\
\hline$B R C A 1$ & 17:41245984-41245987 & c.1561_1564delGCAGinsTAAA & p.Ala521* & p.Asp522Asn, p.Ala521Glu, p.Ala521Ser \\
\hline BRCA1 & $17: 41244552-41244553$ & c.2995_2996delCTinsTA & p.Leu999* & p.Leu999GIn, p.= \\
\hline TP53 & $17: 7578486-7578488$ & c.442_444delGATinsTGA & p.Asp $148^{*}$ & p.Asp148Glu, p.Asp148Gly, p.Asp148Tyr \\
\hline TP53 & $17: 7578286-7578287$ & c.562_563delCTinsTA & p.Leu $188^{*}$ & p.Leu188Gln, p.= \\
\hline \multicolumn{5}{|c|}{ Rescued protein-truncating effect } \\
\hline TP53 & 17:7579366-7579368 & c.319_321delTACinsCAA & p.Tyr107Gln & p.Tyr107*, p.Tyr107His \\
\hline TP53 & $17: 7578535-7578536$ & c.394_395delAAinsTG & p.Lys132Trp & p.Lys132Arg, p.Lys132* \\
\hline TP53 & $17: 7578433-7578434$ & c.496_497delTCinsGG & p.Ser166Gly & p.Ser166*, p.Ser166Ala \\
\hline TP53 & $17: 7578426-7578431$ & c.499_503delinsTACCT & p. GIn167_His168delinsTyrLeu & p.His168Leu, p.GIn167His, p.Gln167* \\
\hline TP53 & $17: 7578210-7578212$ & c.637_639delCGAinsTGG & p.Arg213Trp & p. $=$, p.Arg213* \\
\hline TP53 & 17:7577508-7577509 & c.772_773delGAinsTT & p.Glu258Leu & p.Glu258Val, p.Glu258* \\
\hline
\end{tabular}


NA12878 myeloid neoplasm panel dataset (Illumina TruSight myeloid panel) was retrieved from Illumina BaseSpace [20]. GATK HaplotypeCaller version 3.6 [7] and SAMtools version 1.3 [12] with default parameters were used for variant calling. Concordance comparison of variant calls was assisted by vcfeval tool of RTG Tools version 3.6.2 [21].

\section{Germline complex indel detection in breast and/or ovarian cancers}

A total of 239 clinically high-risk breast and/or ovarian cancer patients from Hong Kong Hereditary and High Risk Breast Cancer Programme were selected for this study. Patients were recruited from January 18, 2007 to December 2, 2015 according to previously described criteria [6]. Three patients carrying germline complex indel mutation in either BRCA1 or BRCA2 (confirmed by Sanger sequencing) were regarded as positive controls. Another 236 patients either carrying pathogenic mutation other than complex indel or not carrying any pathogenic mutation in BRCA1 and BRCA2 (confirmed by full gene Sanger sequencing) were regarded as negative controls. Complex indel detection by INDELseek was performed on BWA-MEM (version 0.7.7) alignments of MiSeq NGS data of full BRCA1 and BRCA2 genes [6]. The definition of full $B R C A 1$ and $B R C A 2$ genes, sequencing methods, analysis methods and partial results were reported previously [6].

\section{Somatic complex indel detection in myeloid neoplasms} Twenty-two archival DNA samples were retrieved in Hong Kong Sanatorium \& Hospital from May 12, 2014 to February 3, 2016. Five of the DNA samples carried somatic pathogenic CALR or JAK2 complex indels and were regarded as positive controls. Remaining seventeen DNA samples of healthy adults with normal complete blood profile were regarded as negative controls as described [16]. Ten core-binding factor leukemia DNA samples were retrieved from Queen Mary Hospital, Hong Kong from January 2003 to December 2014 as a discovery cohort of KIT exon 8 mutations. A total of 32 DNA samples were screened by MiSeq sequencing of a 54-gene myeloid NGS gene panel as described [16, 17]. Complex indel detection by INDELseek was performed on BWA-MEM (version 0.7.7) alignments of MiSeq NGS data of CALR, JAK2 and KIT (exon 8 only).

\section{In silico engineering of known complex indels to real NGS data}

Known complex indels were collected based on VCF files from COSMIC v71 release [22] and dbSNP b146 release [23]. MutationEngineer was developed to engineer mutation into described real NGS data. Input is the variant of interest and NGS read alignments (VCF and SAM formats, respectively) and output is the engineered read alignments
(SAM format) for conversion to FASTQ sequencing reads. Variant allele frequency of complex indel was engineered to be $100 \%$. Each complex indel was engineered as a separate set of FASTQ reads, which were analyzed in the same way as real NGS data. Variants were annotated using Variant Effect Predictor version 75 [24]. Semi-simulation was performed on a Cray XC30 supercomputer.

\section{Orthogonal validation}

$B R C A 1$ and BRCA2 complex indels were confirmed by conventional PCR and Sanger sequencing [6]. CALR and JAK2 complex indels were confirmed by conventional PCR and Sanger sequencing or conventional PCR fragment analysis $[5,16]$. KIT exon 8 complex indels were confirmed by conventional PCR fragment analysis [16] or microfluidic PCR followed by MiSeq sequencing [25]. The primers used in these validation studies were different from those used in the original NGS datasets (Additional file 1: Table S3).

\section{Reference sequences}

Human reference genome sequence: GRCh37/hg19, BRCA1: NM_007294.3, BRCA2: NM_000059.3, TP53: NM_000546.4, PTEN: NM_000314.4, CALR: NM_004 343.3, KIT: NM_000222.2 and JAK2: NM_004972.3. Variants were described according to the recommendations of Human Genome Variation Society (HGVS) [26]. Variant descriptions were checked by Mutalyzer Name Checker [26].

\section{Availability and requirements}

The authors declare that the data supporting the findings of this study are available within the article and its supplementary information files. Primary sequencing data of clinical samples are available on request from the corresponding author ESKM. The sequencing data are not publicly available due to them containing information that could compromise research participant privacy or consent.

Project name: INDELseek

Project home page: https://github.com/tommyau/ indelseek/

Operating system(s): Unix-like (Linux, Mac OS X)

Programming language: Perl

Other requirements: SAMtools 1.3 or higher

License: free for non-profit and academic use.

\section{Additional files}

Additional file 1: Table S1. Putative complex indels curated from GIAB high-confidence variant calls $(n=160)$. Table S2. Closely spaced SNV in trans curated from GIAB high-confidence variant calls $(n=26)$. Table S3. Primer sequences for orthogonal validation. (PDF $564 \mathrm{~kb}$ )

Additional file 2: Figures S1-S14. Complex indels detected in samples 1-14 and orthogonal validation. (PDF $1637 \mathrm{~kb}$ ) 


\section{Acknowledgements}

The authors thank all clinicians who submitted patient samples for diagnostic study.

\section{Funding}

This work was supported by the Hong Kong Hereditary Breast Cancer Family Registry, Dr. Ellen Li Charitable Foundation, and the S K Yee Medical Foundation. AYHL is the Li Shu Fan Medical Foundation Professor in Haematology and has received funding from its endowment.

\section{Authors' contributions}

ESKM and TLC conceived the study. AK, ESKM and AYHL collected samples. CHA, TLC and ESKM analyzed the data. CHA developed the bioinformatics software and drafted the manuscript. All authors read and approved the final manuscript.

\section{Competing interests}

The authors declare that they have no competing interests.

\section{Consent for publication}

Not applicable.

\section{Ethics approval and consent to participate}

The study involving breast and/or ovarian cancers was approved by the Institutional Review Board of the University of Hong Kong/Hospital Authority West Cluster and other contributing hospitals in Hong Kong. The study involving myeloid neoplasms was approved by the Research Ethics Committee of Hong Kong Sanatorium \& Hospital (reference number: REC-2015-02) and The University of Hong Kong/Hong Kong West Cluster (reference number: UW 14-639). All participants gave written informed consent; with the exception that informed consent was not needed for the use of pre-existing de-identified archival DNA samples (22 samples from Hong Kong Sanatorium \& Hospital and 10 samples from Queen Mary Hospital for the study involving myeloid neoplasms).

\section{Author details}

'Division of Molecular Pathology, Department of Pathology, Hong Kong Sanatorium \& Hospital, Happy Valley, Hong Kong SAR. ${ }^{2}$ Department of Medicine, The University of Hong Kong, Pok Fu Lam, Hong Kong SAR. ${ }^{3}$ Department of Surgery, The University of Hong Kong, Pok Fu Lam, Hong Kong SAR. ${ }^{4}$ Department of Surgery and Cancer Genetics Center, Hong Kong Sanatorium \& Hospital, Happy Valley, Hong Kong SAR. ${ }^{5}$ Hong Kong Hereditary Breast Cancer Family Registry, Shau Kei Wan, Hong Kong SAR.

\section{Received: 5 August 2016 Accepted: 21 December 2016}

\section{Published online: 05 January 2017}

\section{References}

1. den Dunnen JT, Antonarakis SE. Mutation nomenclature extensions and suggestions to describe complex mutations: a discussion. Hum Mutat. 2000; 15(1):7-12.

2. Howlett NG, Taniguchi T, Olson S, Cox B, Waisfisz Q, De Die-Smulders C, et al. Biallelic inactivation of BRCA2 in Fanconi anemia. Science. 2002; 297(5581):606-9.

3. Ye K, Wang J, Jayasinghe R, Lameijer E, McMichael JF, Ning J, et al. Systematic discovery of complex insertions and deletions in human cancers. Nat Med. 2016;22(1):97-104.

4. Lek M, Karczewski K, Minikel EV, Samocha KE, Banks E, Fennell T, et al. Analysis of protein-coding genetic variation in 60,706 humans. Nature. 2016; 536(7616):285-91.

5. Klampfl T, Gisslinger H, Harutyunyan AS, Nivarthi H, Rumi E, Milosevic JD, et al. Somatic mutations of calreticulin in myeloproliferative neoplasms. N Engl J Med. 2013;369(25):2379-90.

6. Kwong A, Shin VY, Au CH, Law FBF, Ho DN, Ip BK, et al. Detection of germline mutation in hereditary breast and/or ovarian cancers by next-generation sequencing on a four-gene panel. J Mol Diagn. 2016;18(4):580-94.

7. DePristo MA, Banks E, Poplin R, Garimella KV, Maguire JR, Hartl C, et al. A framework for variation discovery and genotyping using next-generation DNA sequencing data. Nat Genet. 2011;43(5):491-8.
8. Koboldt DC, Zhang Q, Larson DE, Shen D, McLellan MD, Lin L, et al. VarScan 2: somatic mutation and copy number alteration discovery in cancer by exome sequencing. Genome Res. 2012;22(3):568-76.

9. Kadri S, Zhen CJ, Wurst MN, Long BC, Jiang Z, Wang YL, et al. Amplicon indel hunter is a novel bioinformatics tool to detect large somatic insertion/ deletion mutations in amplicon-based next-generation sequencing data. J Mol Diagn. 2015;17(6):635-43.

10. Yang R, Nelson AC, Henzler C, Thyagarajan B, Silverstein KAT. Scanlndel: a hybrid framework for indel detection via gapped alignment, split reads and de novo assembly. Genome Med. 2015;7:127.

11. Wei L, Liu LT, Conroy JR, Hu Q, Conroy JM, Morrison CD, et al. MAC: identifying and correcting annotation for multi-nucleotide variations. BMC Genomics. 2015;16:569.

12. Li H, Handsaker B, Wysoker A, Fennell T, Ruan J, Homer N, et al. The sequence alignment/map format and SAMtools. Bioinformatics. 2009; 25(16):2078-9.

13. Danecek P, Auton A, Abecasis G, Albers CA, Banks E, DePristo MA, et al. The variant call format and VCFtools. Bioinformatics. 2011;27(15):2156-8.

14. Zook JM, Chapman B, Wang J, Mittelman D, Hofmann O, Hide W, et al. Integrating human sequence data sets provides a resource of benchmark SNP and indel genotype calls. Nat Biotechnol. 2014;32(3):246-51.

15. Pruitt KD, Harrow J, Harte RA, Wallin C, Diekhans M, Maglott DR, et al. The consensus coding sequence (CCDS) project: Identifying a common proteincoding gene set for the human and mouse genomes. Genome Res. 2009; 19(7):1316-23.

16. Au CH, Wa A, Ho DN, Chan TL, Ma ESK. Clinical evaluation of panel testing by next-generation sequencing (NGS) for gene mutations in myeloid neoplasms. Diagn Pathol. 2016;11:11.

17. Cher CY, Leung GMK, Au CH, Chan TL, Ma ESK, Sim JPY, et al. Nextgeneration sequencing with a myeloid gene panel in core-binding factor AML showed KIT activation loop and TET2 mutations predictive of outcome. Blood Cancer J. 2016;6(7):e442.

18. Quinlan AR, Hall IM. BEDTools: a flexible suite of utilities for comparing genomic features. Bioinformatics. 2010;26(6):841-2.

19. Eberle MA, Fritzilas E, Krusche P, Kallberg M, Moore BL, Bekritsky MA, et al. A reference dataset of 5.4 million phased human variants validated by genetic inheritance from sequencing a three-generation 17-member pedigree. bioRxiv. 2016. Retrieved from http://biorxiv.org/content/early/2016/08/02/055541.

20. Illumina BaseSpace. https://basespace.illumina.com/. Accessed 7 Mar 2015.

21. Real Time Genomics RTG Tools. http://realtimegenomics.com/products/rtg-tools/. Accessed 11 Mar 2016.

22. Sherry ST, Ward MH, Kholodov M, Baker J, Phan L, Smigielski EM, et al. dbSNP: the NCBI database of genetic variation. Nucleic Acids Res. 2001; 29(1):308-11.

23. Forbes SA, Beare D, Gunasekaran P, Leung K, Bindal N, Boutselakis H, et al. COSMIC: exploring the world's knowledge of somatic mutations in human cancer. Nucleic Acids Res. 2015;43(Database issue):805.

24. McLaren W, Pritchard B, Rios D, Chen Y, Flicek P, Cunningham F. Deriving the consequences of genomic variants with the Ensembl API and SNP Effect Predictor. Bioinformatics. 2010;26(16):2069-70.

25. Lange V, Böhme I, Hofmann J, Lang K, Sauter J, Schöne B, et al. Costefficient high-throughput HLA typing by MiSeq amplicon sequencing. BMC Genomics. 2014;15:63.

26. den Dunnen JT. Sequence variant descriptions: HGVS nomenclature and mutalyzer. Curr Protoc Hum Genet. 2016;90:7. 13.19. 